\title{
EVERY $G$-MODULE IS A SUBMODULE OF A DIRECT SUM OF CYCLICS
}

\author{
ANDY R. MAGID
}

(Communicated by Ronald M. Solomon)

\begin{abstract}
Let $G$ be a group and $V$ a finite-dimensional complex $G$-module. It is shown that $G$ is (isomorphic to) a submodule of a direct sum $W_{1} \oplus \cdots \oplus W_{s}$ where each $W_{i}$ is a cyclic finite-dimensional complex $G$-module. If $G$ is an analytic (respectively algebraic) group and $V$ is an analytic (respectively rational) module then the $W_{i}$ can be taken to be analytic (respectively rational).
\end{abstract}

\section{INTRODUCTION}

Let $G$ be a complex analytic group. As Singer has pointed out, [7, Corollary 2.15, p. 38] essentially asserts that every (finite-dimensional, analytic) $G$ module is a submodule of a direct sum of cyclic (finite-dimensional, analytic) $G$-modules. Unfortunately, the proof provided does not establish the assertion.

One can raise the same question-is every module a submodule of a direct sum of cyclic modules-for any group, and indeed for any ring. In this paper, attention will be restricted to modules finite-dimensional over an algebraically closed characteristic zero field $k$. It is easy to exhibit examples of rings for which the question has a negative answer (see Example 4.1 below). For groups, however, the answer turns out always to be positive; it is the purpose of this note to establish this result.

If $V$ is a module for the group $G$, there is a natural homomorphism $G \rightarrow$ $\operatorname{GL}(V)$. Let $\bar{G}$ be the Zariski closure of its image. Then $V$ is also a rational $\bar{G}$-module, while any rational $\bar{G}$-module is by restriction a $G$-module. This observation reduces us to the case of linear algebraic groups. Since simple modules are cyclic, semisimple modules are direct sums of cyclic modules. So the key case to consider is that of a unipotent algebraic group. This is done in $\S 2$ below. In $\S 3$ the extension to the case of general algebraic groups, analytic groups, and groups in general is given. Section 4 is devoted to examples and remarks.

Received by the editors December 28, 1990 and, in revised form, April 17, 1991. 1980 Mathematics Subject Classification (1985 Revision). Primary 20C07, 20 G05. Key words and phrases. Algebraic group, rational module. 


\section{UNIPOTENT GROUPS}

Let $U$ be a unipotent algebraic group over the (algebraically closed characteristic zero) field $k$. We recall some facts about the structure of the coordinate ring $k[U]$ as an algebra and as a $U$-module; the references are [6] and [8]. The notation to be introduced is to remain in force throughout this section.

First, we recall the structure of $k[U]$ as a $U$-module. $U$ acts on $k[U]$ on the left by $(x \cdot f)(y)=f(y x)$ and on the right by $(f \cdot x)(y)=f(x y)$. The one-dimensional trivial $U$-module $k$ lies in $k[U]$ as the constants, and $k[U]$, as a left $U$-module, is the injective hull of $k$ in the category of rational $U$-modules [2]; we use $E(U)$ to denote $k[U]$ so regarded. We define an ascending chain of submodules $E_{i}(U)$ of $E(U)$ by $E_{-1}(U)=\{0\}$ and $E_{i+1}(U)=$ $\left\{f \mid f \cdot x-f \in E_{i}(U) \forall x \in H\right\}$. When there is no confusion, we write $E_{i}$ for $E_{i}(U)$.

The lower central series of $U$ is given by $C^{1} U=U$ and $C^{p+1} U=\left(U, C^{p} U\right)$. If $x_{1}, \ldots, x_{d}$ in $U$ are such that $\left\{x_{i} C^{2} U\right\}$ is a $k$-basis of $C^{1} U / C^{2} U$, then $x_{1}, \ldots, x_{d}$ generate $U$. Fix such a minimal generating set, and let $X_{p}$ denote the endomorphism of $E(U)$ given by $X_{i}(f)=f \cdot x_{i}^{-1}-f$. Then

$$
E_{p}(U)=\left\{f \in U \mid m \cdot f=0 \forall m \text { a monomial in the } X_{i} \text { of weight }>p\right\} .
$$

As a $k$-algebra, $k[U]$ is a polynomial ring. We choose any rational isomorphism $\operatorname{Lie}(U) \rightarrow U$ and any $k$-basis of $\operatorname{Lie}(U)$ to give polynomial generators. To link the algebra structure and the module structure, we make the choice as follows: let $\mathscr{C}^{i} \operatorname{Lie}(U)$ denote the lower central series of $\operatorname{Lie}(U)$, where $\mathscr{C}^{1} \operatorname{Lie}(U)=\operatorname{Lie}(U)$ and $\mathscr{C}^{i+1} \operatorname{Lie}(U)=\left[\operatorname{Lie}(U), \mathscr{C}^{i} \operatorname{Lie}(U)\right]$. Let elements $Y_{i j} \in \mathscr{C}^{i} \operatorname{Lie}(U)$ be chosen so that $\left\{Y_{i j}+\mathscr{C}^{i} \operatorname{Lie}(U)\right\}$ is a basis for $\mathscr{C}^{i} \operatorname{Lie}(U) / \mathscr{C}^{i+1} \operatorname{Lie}(U)$. Then $\left\{Y_{i j}\right\}$ is a basis of $\operatorname{Lie}(U)$. Let $\left\{b_{i j}\right\}$ be the dual basis, and define $f_{i j} \in k[U]$ by $f_{i j}=b_{i j} \circ \log$. Then $k[U]=k\left[\left\{f_{i j}\right\}\right]$, and for each $p, E_{p}(U)$ has for a basis the set of all products

$$
\prod f_{i_{j}}^{n_{i j}} \quad \text { such that } \quad \sum i n_{i j} \leq p
$$

(see [6, Theorem 2.5, p. 54]). In other words, if we regard $k[U]$ as a graded polynomial ring where the generator $f_{i j}$ has weight $i$, then $E_{p}$ is spanned by the monomials of weight $p$ or less.

Every $U$-endomorphism of $E(U)$ can be expressed as a formal power series in $X_{1}, \ldots, X_{d}[5$, Theorem 1.6, p. 327]. This applies in particular to the $U$ invariant derivations of $k[U]$, namely to $\operatorname{Lie}(U)$. We want to consider $E(U)$ as a module over the universal enveloping algebra $\mathscr{U}(\operatorname{Lie}(U))$, and for our purposes it will be sufficient to consider its image $\mathscr{A}$ in $\operatorname{End}_{U}(E(U))$. We have a natural filtration on $\operatorname{End}_{U}(E(U))$ given by the degree of the leading monomial in a power series expansion of an endomorphism; that is, by the powers of the augmentation ideal of $k\left[X_{1}, \ldots, X_{d}\right]$ (which by [5, Theorem 1.5 , p. 327] consists of the endomorphisms vanishing on constants). This induces a filtration on $\mathscr{A}$. We isolate the main fact about this filtration in the following lemma.

Lemma 2.1. Let $\mathscr{A}$ denote the subalgebra of $\operatorname{End}_{U}(E(U))$ generated by $\operatorname{Lie}(U)$, and let I denote the ideal of $\mathscr{A}$ consisting of all endomorphisms vanishing on $k$. If $T \in I^{p}$ and $f \in E_{q}(U)$, then $T \cdot f \in E_{q-p}(U)$. 
Proof. $T$ can be expressed as a formal power series in $X_{1}, \ldots, X_{d}$. Since $T \in I^{p}$, the monomials in $T$ are of weight $p$ or higher. Let $m$ be such a monomial. If $n$ is any monomial of weight $>q-p$, then $n \cdot m$ is of weight $q$ or higher. Thus if $f \in E_{q}(U)$, we have $n \cdot m \cdot f=0$. This holds for every monomial of weight $>q-p$, so we conclude that $m \cdot f \in E_{q}(U)$. It follows that $T \cdot f \in E_{q}(U)$.

We will want to apply Lemma 2.1 to expressions involving elements of $\operatorname{Lie}(U)$. To that end, we recall that inside the universal enveloping algebra of a free Lie algebra (which is itself a free algebra), the degree of a Lie monomial in the Lie algebra is equal to its degree in the free algebra [9, Theorem 5.8, p. 323]. Since $\mathscr{C}^{p} \operatorname{Lie}(U)$ is spanned by Lie monomials of weight $p$, this means the following in terms of the above filtration on $\mathscr{A}$.

Corollary 2.2. In the notation of (1.1), if $D \in \mathscr{C}^{p} \operatorname{Lie}(U)$, then $D \in I^{p}$.

Next, we prove the main result of this section in the case that $U$ has dimension one.

Lemma 2.3. Assume $\operatorname{dim}(U)=1$, and let $f$ denote the additive character generating $k[U]$. Then $E_{p}(U)$ is cyclic, generated by $f^{p}$.

Proof. Let $x$ denote a generator of $U$. We will write $x^{a}$ for $\exp (a \log x)$. Then $f$ is given by $f\left(x^{a}\right)=a$, so $x \cdot f=f+1$ and $f \cdot x^{-1}=f-1$. Thus $x \cdot f^{q}=(f+1)^{q}$, from which it follows that $\left\{g \mid \operatorname{deg}_{f}(g) \leq p\right\}$ is a $U$-stable submodule of $E(U)$ and also that if $X$ denotes the transformation $X(g)=$ $g \cdot x^{-1}-g$, then the $X^{r}\left(f^{s}\right)$ has maximal degree $s-r$ in $f$. Thus the set of elements of $E(U)$ annihilated by all $X^{r}$ for $r>p$ is spanned by $\left\{f^{i} \mid i \leq p\right\}$, so $E_{p}$ is that span. It is an exercise with the binomial theorem to pass from the formula $x \cdot f^{q}=(f+1)^{q}$ (so that $q^{-1}\left(x \cdot f^{q}-f^{q}\right)=f^{q-1}+$ lower degree ) to the conclusion that $f^{p}$ generates $E_{p}(U)$ as a $U$-module.

Now we come to our main result.

Theorem 2.4. Let $U$ be a unipotent algebraic group, and let $p \geq 0$. Then there is an element $g$ in $E(U)$ such that the cyclic $U$-submodule generated by $g$ contains $E_{p}(U)$.

Proof. We will proceed by induction on the dimension of $U$; the case of $\operatorname{dim}(U)=1$ being covered by Lemma 2.3. We use the structure of $E(U)$ recalled above. Assume that $C^{s} U \neq 1$ and $C^{s+1} U=1$, and let $c$ denote the dimension of $C^{s} U=\mathscr{C}^{s} \operatorname{Lie}(U)$. Above, we have selected a basis $Y_{s 1}, \ldots, Y_{s c}$ of $C^{s} U$ and corresponding functions $f_{s_{1}}, \ldots, f_{s_{C}}$. For the rest of this proof, we will also denote $Y_{s_{c}}$ by $D$ and $f_{s_{c}}$ by $f$. Let $Z$ denote the subgroup $\exp (k D)$ of $U ; Z$ is central because $D$ is central in $\operatorname{Lie}(U)$. We form the quotient $\bar{U}=U / Z$. Now $\operatorname{dim}(\bar{U})=\operatorname{dim}(U)-1$, so by induction we may assume that $E_{p}(\bar{U})$ is contained in the cyclic $\bar{U}$-submodule of $E(\bar{U})$ generated by some element $m$, which we assume belongs to $E_{r}(\bar{U})$. As noted above, $E_{p}(U)$ is spanned by the monomials $\prod f_{i_{j}}^{n_{i j}}$, where $\sum i n_{i j} \leq p$. The surjection $U \rightarrow \bar{U}$ induces a surjection $\operatorname{Lie}(U) \rightarrow \operatorname{Lie}(\bar{U})$, which we will denote by overlining. By construction, the set $\left\{\bar{Y}_{i j} \mid 1 \leq i \leq s, 1 \leq j \leq c-1\right\}$ forms a basis of $\operatorname{Lie}(\bar{U})$ compatible with its lower central series (we make the obvious index adjustment if $c=1$ ), and we can define the corresponding functions $\left\{\bar{f}_{i j}\right\}$ on 
$\bar{U}$. The inclusion $k[\bar{U}] \rightarrow k[U]$ carries $\bar{f}_{i j}$ to the corresponding $f_{i j}$. We denote the image of $E_{i}(\bar{U})$ by $\bar{E}_{i}$. Note that $f \in E_{s}(U)$, but $f \notin E_{s-1}(U)$, that $\bar{E}=k[U]^{Z}$, and that $E=\bar{E}[f]=\sum \bar{E} f^{i}$ (polynomial ring). With the above definitions of $\bar{E}_{i}$, the correspondence between the $\bar{f}_{i j}$ and their images in $E(U)$, and the given monomial basis, we have the formula

$$
E_{p}(U)=\sum_{i=0}^{\ell}\left(\bar{E}_{p-i s}\right) f^{i} \quad \text { where } \ell=\sup \{j \mid s j \leq p\} .
$$

Now let $g=m f^{\ell}$. We are going to show that the cyclic submodule generated by $g$ contains $E_{p}$. We recall that $m \in \bar{E}_{r}$ and that $f \in E_{s}$. By (*) above, it suffices to prove that each $\left(\bar{E}_{p-i s}\right) f^{i}$ is contained in the submodule generated by $g$. As above, we let $\mathscr{A}$ be the subalgebra of $\operatorname{End}_{U}(E(U))$ generated by $\operatorname{Lie}(U)$. Its restriction $\overline{\mathscr{A}}$ to $\bar{E}$ is isomorphic to the subalgebra of $\operatorname{End}_{\bar{U}}(E(\bar{U}))$ generated by $\operatorname{Lie}(\bar{U})$. This means that $\bar{E}_{p} \subseteq \overline{\mathscr{A}} \cdot m$. In particular, if $y \in \bar{E}_{p-i s}$, then there is a $T \in \overline{\mathscr{A}}$ such that $T m=y$. Since $m \in \bar{E}_{r}$ and $y \in \bar{E}_{p-i s}$, it follows from Lemma 2.1 that $T \in I^{q}$, where $q \geq r-(p-i s)$. We want to consider the element $h=T D^{l-i}\left(m f^{\ell}\right)$. Since $m$ lies in $\bar{E}_{\ell-1}=k[U]^{Z}$ and $D$ generates $\operatorname{Lie}(Z), D(m)=0$. Also, $D\left(f^{\ell}\right)=\ell f^{\ell-1}$. So $D^{\ell-i}\left(m f^{\ell}\right)$ is a nonzero constant times $m f^{i}$ and $h$ is a constant multiple of $T\left(m f^{i}\right)$. We can write $T$ as a sum of monomials in the derivations $Y_{i j}$ where $i<s$ or $j<c$. Let $Y$ be such a derivation. Then $Y\left(m f^{i}\right)=Y(m) f^{i}+i m Y(f) f^{i-1}$. Since $f \in E_{s}$ and $Y \in I, Y(f) \in E_{s-1}$, and of course no power of $f$ can appear in $E_{s-1}$. Thus $Y\left(m f^{i}\right)=Y(m) f^{i}+$ terms of lower degree in $f$, and by repeating this argument we discover that $T\left(m f^{i}\right)=T(m) f^{i}+$ terms of lower degree in $f$; that is, $h$ is a constant multiple of $y f^{i}+h^{\prime}$, where the degree in $f$ of $h^{\prime}$ is less than $i$. Since $T \in I^{q}$ for some $q \geq r-(p-i s), T D^{\ell-i}$ belongs to $I^{r-p+\ell s}$. Also, $m f^{\ell}$ belongs to $E_{r+\ell s}$, so $h$ belongs to $E_{(r+\ell s)-(r-p+\ell s)}=E_{p}$. In other words, for each $i, 0 \leq i \leq \ell$, and each $y \in \bar{E}_{p-i s}$, the cyclic submodule $M$ generated by $y$ contains an element $h$ of the form $y f^{i}+h^{\prime}$ that belongs to $\bar{E}_{p}$ and has $\operatorname{deg}_{f}\left(h^{\prime}\right)<i$. Note that since $y f^{i} \in \bar{E}_{p}, h^{\prime}=h-y f^{i} \in \bar{E}_{p}$. These statements also apply when $p$ is replaced by $p-k$. Since $E_{p-k}=$ $\sum_{i}\left(\bar{E}_{(p-k)-i s}\right) f^{i}$, we need only replace $\ell-i$ by the appropriate power $e$ in forming $T D^{e}\left(m f^{\ell}\right)$, which is a constant multiple of $T\left(m f^{i}\right)$. In other words, for each element $y$ of $\bar{E}_{p-k-i s}$, there is an element in $M \cap \bar{E}_{p-k}$ of the form $y f^{i}+h^{\prime}$ where the degree of $h^{\prime}$ in $y$ is less than $i$.

Now we argue inductively on $i$ that $\bar{E}_{p-i s} f^{i} \subseteq M$ for $i=0,1, \ldots, \ell$. For $i=0$, this follows from the fact that $\bar{E}_{p} \subseteq \mathscr{A} \cdot m$. Suppose the inclusion held for all $j<i$, and let $y f^{i} \in \bar{E}_{p-i} f^{i}$. By the above, there is $h \in E_{p} \cap M$ of the form $h=y f^{i}+h^{\prime}$ with $\operatorname{deg}_{f}\left(h^{\prime}\right)<i$. It follows that $h^{\prime}$ belongs to $\sum_{j=0}^{i-1} \bar{E}_{p-j s} f^{j}$, where the sum ranges over $j$ with $s j<i$. By the induction hypothesis, $h^{\prime} \in M$. Thus $y f^{i}=h-h^{\prime} \in M$, so $\bar{E}_{p-i s} f^{i} \subseteq M$, and the proof of Theorem 2.4 is complete.

We observe that the induction step of Theorem 2.4 starts with an element in $E_{r}(\bar{U})$ generating a cyclic submodule containing $E_{p}(\bar{U})$ and producing an element in $E_{r \ell s}(U)$ generating a cyclic submodule containing $E_{p}(U)$, where $\ell$ is 
$\sup \{j \mid s j \leq p\}$ and $s$ is the length of the lower central series of $U$. This produces the following explicit form of Theorem 2.4:

Corollary 2.5. Let $\left\{f_{i j}\right\}$ generate $k[U]$ as in the proof of (2.4). Fix $p \geq 0$, and let $\ell_{i}=\sup \{e \mid i e \leq p\}$. Then $E_{p}(U)$ is contained in the cyclic submodule of $E(U)$ generated by $\prod\left(f_{i j}\right)^{\ell_{i}}$.

Also, since $E(U)$ is the ascending union of its submodules $E_{p}(U)$, we can also restate Theorem 2.4 in the following form.

Corollary 2.6. Let $U$ be a unipotent group. Then any finitely generated submodule of $E(U)$ is contained in a cyclic submodule.

Corollary 2.6 also remains true for prounipotent groups $U$, since any finitely generated submodule of $k[U]$ actually lies inside $k[\bar{U}]$ for some unipotent quotient $\bar{U}$ of $U$. (See [3-5] for the theory of prounipotent groups.) In fact, for the case of free prounipotent groups in the sense of [5, Definition 2.1, p. 83], we have a somewhat stronger statment (see Proposition 2.9 below). The following remarks about Lie algebras will play a role in that proof.

Proposition 2.7. Let $L$ be a (finite-dimensional) Lie algebra, $\mathscr{U}$ its universal enveloping algebra, and $I$ the augmentation ideal of $\mathscr{U}$. Then

(1) If $x \in I^{n}-I^{n+1}$ and $y \in I^{m}-I^{m+1}$, then $x y \in I^{n+m}-I^{n+m+1}$.

(2) $\left\{\bar{x} \in \mathscr{U} / i^{n+1} \mid I \cdot \bar{x}=0\right\}=I^{n} / I^{n+1}$.

Proof. The associated graded ring $\operatorname{gr}_{I}(\mathscr{U})=\bigoplus I^{p} / I^{p+1}$ is canonically isomorphic to the universal enveloping algebra of $\operatorname{gr}(L)=\bigoplus \mathscr{C}^{p} L / \mathscr{C}^{p+1} L$ [11] and hence is an integral domain. Thus (1) obtains. For (2), let $\bar{x}=x+I^{n+1}$ belong to the left-hand side. Assume $x \in I^{m}-I^{m+1}$ for $m=n-k$. If $k>0$, choose $y \in I^{k}-I^{k+1}$. Then $y x$ belongs to $I^{m+k}-I^{m+k+1}=I^{n}-$ $I^{n+1}$, so $y \bar{x} \neq 0$. Thus $k=0$, so $x \in I^{n}$, and we conclude that the left-hand side of (2) is contained on the right. The reverse inclusion is obvious so (2) is obtained.

This applies to unipotent groups as follows.

Lemma 2.8. Let $U$ be a unipotent group. Then

$$
\left(E_{p}(U) / E_{p-1}(U)\right)^{*}=\left(E_{p}(U)\right)^{*}
$$

that is, the socle of $E_{p}^{*}$ is $\left(E_{p} / E_{p-1}\right)^{*}$ and $E_{p-1}$ is the Fratinni submodule of $E_{p}$.

Proof. Let $L$ denote $\operatorname{Lie}(U), \mathscr{U}$ denote the universal enveloping algebra of $L$, and $I$ denote the augmentation ideal of $\mathscr{U}$. Then $E_{p}=\left(\mathscr{U} / I^{p+1}\right)^{*}$ as an $L$-module [4, Example 1.8, p. 535], so $E_{p}^{*}=\mathscr{U} / I^{p+1}$ as an $L$-module. For any $U$-module $M$ regarded as a $\mathscr{U}$-module, we have $M^{U}=\{x \in M \mid I x=0\}$ so that Lemma 2.8 follows from the second part of Proposition 2.7.

Lemma 2.8 also applies to finitely generated prounipotent groups, since in that case all the modules involved factor through unipotent quotient groups.

For any unipotent (or prounipotent) group $U$, the module $E_{p} / E_{p-1}$ has trivial $U$-action, as does the isomorphic (by Lemma 2.8) module $\left(E_{p}^{*}\right)^{U}$. This suggests trying to extend $E_{p}^{*}$ by $E_{p}$ by patching the socle of the former to the cosocle of the latter. As we shall now see, this can be done in the case of 
the free prounipotent group, and the extension turns out to be a cyclic module containing $E_{p}$.

Proposition 2.9. Let $F$ be a free prounipotent group on $d$ generators. Then there exists an $F$-submodule $M$ of $E(F)$ containing $E_{p}$ such that $M / E_{p-1}$ is isomorphic to $E_{p}^{*}$. Moreover, $M$ is cyclic.

Proof. $E / E_{p-1}$ is the direct sum of $n$ copies of $E$, where $n=$ $\operatorname{dim}\left(\left(E / E_{p-1}\right)^{F}\right)\left[5\right.$, Lemma 1.3.1, p. 326]. Moreover, $\left(E / E_{p-1}\right)^{F}=E_{p} / E_{p-1}$ [5, Lemma 1.3.3, p. 326]. By Lemma 2.8, $\left(E_{P}^{*}\right)^{F}=\left(E_{p} / E_{p-1}\right)^{*}$. We choose an isomorphism $\left(E_{p} / E_{p-1}\right)^{*} \rightarrow E_{p} / E_{p-1}$, which then also embeds the source into the injective module $E / E_{p-1}$ and hence extends to a homomorphism $h: E_{p}^{*} \rightarrow E / E_{p-1}$, which is a monomorphism since it is injective on the socle $\left(E_{P}^{*}\right)^{F}$ of $E_{p}^{*}$. Let $M$ be the inverse image in $E$ of $h\left(E_{p}^{*}\right)$. Then $M$ contains $E_{p-1}$, and $M / E_{p-1}=E_{p}^{*}$. Let $\bar{M}$ be any semisimple quotient of $M$. (That is, quotient with trivial $F$-action.) Let $\bar{E}_{p}$ be the image of $E_{p}$ in $\bar{M}$. Since this is a semi-simple quotient of $E_{p}$, Lemma 2.8 implies that $E_{p-1}$ must have 0 image in $\bar{E}_{p}$, so $M / E_{p-1}=E_{p}^{*}$ maps onto $\bar{M}$. The socle of $E_{p}$ is $E_{0}=k$, so $k$ is the cosocle of $E_{p}^{*}$. If the cosocle of a module is cyclic, so is the module (the kernel of the canonical projection onto the cosocle is the Fratinni submodule), and hence $E_{p}^{*}$ is cyclic. But then its homomorphic image $\bar{M}$ is also cyclic. It then follows that the cosocle of $M$ is cyclic, whence $M$ itself is cyclic.

The referee has pointed out that when $U$ is the unipotent radical of a Borel subgroup $B$ of a semisimple, simply connected group $G$, then $k[U]$ is known to be the directed union of B-submodules that are obtained from irreducible $G$-modules (actually Steinberg modules) tensored with one-dimensional $B$-modules [1, 5.5, p. 107]. Since irreducible $G$-modules are $U$-cyclic (generated by highest weight vectors) and one-dimensional $B$-modules are $U$-trivial, it follows that $k[U]$ is the directed union of cyclic $U$-submodules. Actually, [1] deals with the case of positive characteristic, but, as the referee pointed out, essentially the same arguments apply in characteristic zero (and provide a rapid proof of Corollary 2.6 above in this special case). We want to record here the additional case of positive characteristic.

Proposition 2.10. Assume that $k$ is an algebraically closed field of positive characteristic. Let $G$ be a connected, simply connected, semisimple algebraic group over $k$, and let $U$ be the unipotent radical of a Borel subgroup of $G$. Then every finite dimensional $U$-submodule of $k[U]$ is contained in in a cyclic submodule.

\section{General Groups}

First we consider the case of linear algebraic groups.

We will need to recall the structure of injective rational modules over such groups $G$ [2]: any such is a direct sum of indecomposable injective modules, which in turn have the form $E_{G}(V)$, the $G$-injective hull of the simple $G$ module $V$. If $G=U \cdot P$, where $U$ is the unipotent radical of $G$ and $P$ is a maximal reductive subgroup, then $E(V)=k[G]^{P} \otimes V$.

Theorem 3.1. Let $G$ be a linear algebraic group over $k$, and let $V$ be a finitedimensional rational G-module. Then there are cyclic (finite-dimensional, 
rational) G-modules $W_{1}, \ldots, W_{d}$ such that

$$
V \subseteq \bigoplus_{i=1}^{d} W_{i}
$$

Proof. We consider the injective hull $E(V)$; this is a direct sum $E(V)=$ $\bigoplus E\left(X_{i}\right)$, where $\oplus X_{i}$ is the socle of $V$. It suffices to prove that the projection of $V$ to $E\left(X_{i}\right)$ is contained in a cyclic submodule, and hence we may assume that $V$ has a simple socle $X$, so $V$ is contained in $E_{G}(X)$.

Thus we need to prove that if $X$ is a simple $G$-module and $V$ is a finitely generated submodule of $E_{G}(X)$, then $V$ is contained in a cyclic submodule. As above, we identify $E(X)$ with $k[G]^{P} \otimes X$, where $P$ is a maximal reductive subgroup of $G$. Let $U$ be the unipotent radical of $G$. As a left $U$-module, $k[G]^{P}$ is isomorphic to $k[U]$ via restriction of functions. As a $U$-module, $k[G]^{P} \otimes X=k[U]^{(n)}$ (direct sum), where $\operatorname{dim}(X)=n$. It follows that there is a $p$ such that $V$ is contained in $E_{p}(U) \otimes X$. We can also regard $E_{p}(U)$ as the $p$ th term in the ascending socle series of $k[G]^{P}$, from which it follows that $E_{p}(U)$ is a $G$-submodule. Now choose, by Theorem $2.4, h \in k[U]$ such that the cyclic $U$-submodule generated by $h$ contains $E_{p}(U)$, and let $g \in k[G]^{P}$ restrict to $h$. Choose $x \in X$ nonzero, and let $M$ be the cyclic $G$-submodule of $E_{G}(X)$ generated by $g \otimes X$. Since $U$ acts trivially on $X, M$ contains the span of $U(g \otimes x)=(U g) \otimes x$, which in turn contains $E_{p}(U) \otimes x$. Thus $M$ also contains $G\left(E_{p}(U) \otimes x\right)=E_{p}(U) \otimes G x$ (recall that $G E_{p}(U)=E_{p}(U)$, since $E_{p}(U)$ is a $G$-module) and hence also its span $E_{p}(U) \otimes X$. So $M$ also contains $V$, and then the theorem follows.

(The author is grateful to E. Cline for help with Theorem 3.1.)

We now extend Theorem 3.1 to analytic groups and abstract groups. These results are corollaries of Theorem 3.1 and some observations about Zariski density.

Corollary 3.2. Let $H$ be a complex analytic group and $V$ a finite-dimensional analytic $H$-module. Then there are cyclic analytic $H$-modules $W_{1}, \ldots, W_{d}$ such that $V \subseteq \bigoplus W_{i}$.

Proof. Let $G$ denote the Zariski closure of $H$ in $\mathrm{GL}(V)$. We construct the cyclic $G$-modules $W_{1}, \ldots, W_{d}$ and the embedding $V \rightarrow \bigoplus W_{i}$ by Theorem 3.1. The $W_{i}$ are obviously also analytic $H$-modules by restriction. We claim that $W_{i}$ is also $H$-cyclic; for suppose that $w_{i}$ is a $G$-generator of $W_{i}$ but that the span $X$ of $H w_{i}$ is a proper subspace of $W_{i}$. Choose a nonzero linear functional $\phi$ on $W_{i}$ vanishing on $X$. Then define $f$ on $G$ by $f(g)=\phi\left(g w_{i}\right)$. Then $f$ is a rational function on $G$ vanishing on the Zariski dense subgroup $H$, so $f$ vanishes on $G$. This implies that $\phi$ is zero. This contradiction then implies that $X=W_{i}$ and hence that $W_{i}$ is $H$-cyclic.

The only place that analyticity was used in Corollary 3.2 was to observe that rational $G$-modules are analytic $H$-modules by restriction. As a consequence, the proof for Corollary 3.2 also implies the following for the general case.

Corollary 3.3. Let $\Gamma$ be a group and $V$ a finite-dimensional $k[\Gamma]-$ module. Then there are cyclic $\Gamma$-modules $W_{1}, \ldots, W_{d}$ such that $V \subseteq \bigoplus W_{i}$.

(The author is grateful to A. Lubotzky for pointing out the above corollaries.) 


\section{EXAMPLES}

Our first example shows that there are (finite-dimensional, commutative) $k$ algebras that have modules finite-dimensional over $k$ that are not embeddable in direct sums of cyclic modules (showing that Corollary 3.3 cannot be extended to rings).

Example 4.1. Let $R=k[x, y] /(x, y)^{2}=k[\bar{x}, \bar{y}]$, and let $R^{*}=\operatorname{Hom}_{k}(R, k)$. We will show that the $R$-module $R^{*}$ cannot be embedded in a direct sum of cyclic $R$-modules.

First we determine the cyclic $R$-modules. Ideals of $R$ correspond to ideals $I$ of $k[x, y]$ containing $(x, y)^{2}$, and these are of the form $(x, y)^{2},(x, y)^{2}+$ $(f)$, where $f=a x+b y$ is linear and $(x, y)$. The corresponding cyclic modules $R / I$ are then $R, k[(\bar{x}), \bar{y}] /(a \bar{x}+b \bar{y})$, and $k$, which have $k$ dimension 3,2 , and 1 respectively. Now $R^{*}$ has a basis $\hat{1}, \hat{x}$, and $\hat{y}$ dual to the basis $\overline{1}$, $\bar{x}, \bar{y}$ of $R$, and the $R$ action is $\bar{x} \hat{x}=\hat{1}, \bar{y} \hat{y}=\hat{1}$, and the mixed products 0 . It follows that $R^{*}$ is an essential extension of its submodule $k \hat{1}$. Now suppose $M$ is a cyclic $R$-module and $\phi: R^{*} \rightarrow M$ is an $R$-homomorphism. If $M$ has dimension 3 and $\phi$ is injective then $\phi$ is an isomorphism, which is impossible (the socle of $R$ has dimension 2, and that of $R^{*}$ has dimension 1 ), so either $M$ has dimension 2 or less, or $\phi$ is not injective. In either case, $\operatorname{Ker}(\phi)$ is nonzero, so $\phi(\hat{1})=0$. Since every homomorphism from $R^{*}$ to a cyclic module kills $\hat{1}$, there can be no embedding of $R^{*}$ in a direct sum of cyclic $R$-modules.

Our next example illustrates Corollary 2.5 in the case of the Heisenberg group.

Example 4.2. The Heisenberg group $U$ is the group of $3 \times 3$ upper triangular unipotent matrices. A typical element of $U$ is the matrix

$$
\left[\begin{array}{lll}
1 & a & c \\
0 & 1 & b \\
0 & 0 & 1
\end{array}\right]
$$

which we will denote by the triple $(c, a, b)$. Then $k[U]$ is the polynomial ring $k[x, y, z]$, where $x(c, a, b)=a, y(c, a, b)=b$, and $z(c, a, b)=c . U$ acts on $k[U]$ by $g \cdot x=x+x(g), g \cdot y=y+y(g)$, and $g \cdot z=z+z(g)+y(g) x$.

The Lie algebra of $U$ can be identified with the Lie algebra $L$ of strictly upper triangular matrices. $L$ has a basis

$$
X=\left[\begin{array}{lll}
0 & 1 & 0 \\
0 & 0 & 0 \\
0 & 0 & 0
\end{array}\right], \quad Y=\left[\begin{array}{lll}
0 & 0 & 0 \\
0 & 0 & 1 \\
0 & 0 & 0
\end{array}\right], \quad \text { and } Z=\left[\begin{array}{ccc}
0 & 0 & 1 \\
0 & 0 & 0 \\
0 & 0 & 0
\end{array}\right]
$$

and $X, Y$ give a basis of $\mathscr{C}^{1} L / \mathscr{C}^{2} L$, and $Z$ is a basis of $\mathscr{C}^{2} L$. Since

$$
\exp (a X+b Y+c Z)=I+a X+b Y+c Z+\frac{1}{2} a b Z,
$$

we have, in the notation of $\S 2$ used in Corollary 2.5, $Y_{11}=X, Y_{12}=Y, Y_{21}=$ $Z, f_{11}=x, f_{12}=y$, and $f_{21}=z-\frac{1}{2} x y$; for convenience we denote this last function by $w$. Then $E_{2}$ has basis $w, x^{2}, x y, y^{2}, x, y, 1$ (and we could replace $w$ by $z$ ). By Corollary $2.5, E_{2}$ is contained in the cyclic $U$-module generated by $h=x^{2} y^{2} w \in E_{6}$. 
As we are now going to show, however, we can also exhibit an element of lower degree that also works.

When we interpret $L$ as derivations of $k[U]$, we find that $X \leftrightarrow D_{1}=$ $-(\partial / \partial x+y \partial / \partial z), Y \leftrightarrow D_{2}=-\partial / \partial y$, and $Z \leftrightarrow D_{3}=-\partial / \partial z$.

Let $q=x^{2} y^{2}+z^{2} \in E_{4}$. Then (up to constants) we have

$$
\begin{aligned}
D_{1}^{2}(q) & =y^{2}, \\
D_{1} D_{2}(q) & =x y, \\
D_{2}^{2}(q) & =x^{2}, \\
D_{3}(q) & =z,
\end{aligned}
$$

from which it follows easily that the cyclic $U$-module generated by $q$ contains $E_{2}$.

\section{ACKNOWLEDGMENT}

The author is grateful to Edward T. Cline, Jr. and Alexander Lubotzky for helpful conversations and to Michacl Singer for raising the question. The referee provided the remarks at the end of $\S 2$ covering the case of unipotent radicals of Borel subgroups of simply connected semisimple groups in arbitrary characteristic.

\section{REFERENCES}

1. E. Cline, B. Parshall, and L. Scott, Cohomolgy, hyperalgebars, and representations, J. Algebra 63 (1980), 98-123.

2. In_ Induced modules and affine quotients, Math. Ann. 230 (1977), 1-14.

3. A. Lubotzky and A. Magid, Cohomology of unipotent and prounipotent groups, J. Algebra 74 (1982), 76-95.

4. Cohomology, Poincaré series, and group algebras of unipotent groups, J. Math. 107 (1985), 531-553.

5. __ Free prounipotent groups, J. Algebra 80 (1983), 323-349.

6. A. Magid, Coordinate rings of prounipotent groups, J. Algebra 95 (1985), 46-58.

7. _ Module categories of analytic groups, Cambridge Tracts in Math., vol. 81, Cambridge Univ. Press, New York, 1982.

8. _ Relation modules of prounipotent groups, J. Algebra 109 (1987), 52-68.

9. W. Magnus, A. Karass, and D. Solitar, Combinatorial group theory, 2nd rev. ed., Dover, New York, 1976.

10. C. Nunley and A. Magid, Simple representations of the Heisenberg group, Classical Groups and Related Topic, Contemp. Math., vol. 82, Amer. Math. Soc., Providence, RI, 1989, pp. 89-96.

11. D. Quillen, Rational homotopy theory, Ann. of Math. (2) 90 (1969), 205-295.

12. J. Wang, On the cyclicity and cocyclicity of $G$-modules, Classical Groups and Related Topic, Contemp. Math., vol. 82, Amer. Math. Soc., Providence, RI, 1989, pp. 231-233.

Department of Mathematics, The University of Oklahoma, Norman, Oklahoma 73019 